\title{
Investigation of the Inaccuracy of the MFIE Discretized with the RWG Basis Functions ${ }^{1}$
}

\author{
Özgür ErgüI* and Levent Gürel \\ Department of Electrical and Electronics Engineering \\ Bilkent University \\ Ankara, Turkey \\ (ergul@ee.bilkent.edu.tr, lgurel@bilkent.edu.tr)
}

\section{Introduction}

Electric-field and magnetic-field integral equations are widely used for the numerical solution of the electromagnetic scattering problems by the method of moments (MOM) [1] and the fast multipole method (FMM) [2]. The electric-field integral equation (EFIE) is known to give accurate results with the usage of Rao-Wilton-Glisson (RWG) basis functions for the conducting surfaces with arbitrary planar triangulations [3]. The same functions are also used with the magnetic-field integral equation (MFIE) for the solution of scattering problems involving closed geometries [4]. However, it can be observed that the current distribution and the radar cross section (RCS) obtained by the MFIE does not perfectly match their counterparts obtained by the EFIE, especially when the geometry of the problem includes sharp edges or tips [5]. This paper focuses on the inaccuracies of the solutions obtained by the MFIE as applied to the scattering problems of conducting closed surfaces with planar triangulations. After a thorough investigation, we rule out some of the possible causes of the inaccuracy considered in the literature and we point to the actual reasons behind the inaccuracy of the MFIE.

\section{Inaccuracy of the MFIE}

For closed conducting surfaces, the MFIE can be written as

$$
\bar{J}(\bar{r})-\hat{n} \times \int_{S^{\prime}} d \bar{r}^{\prime} \bar{J}\left(\bar{r}^{\prime}\right) \times \nabla^{\prime} g\left(\tilde{r}, \bar{r}^{\prime}\right)=\hat{n} \times \bar{H}^{i n c}(\bar{r}),
$$

where the observation point approaches to the surface from the outside. Application of the MOM on this formula requires the calculation of the impedance matrix elements with the expression as

$$
Z_{m n}=-\int_{S_{m}} d \bar{r} \bar{t}_{m}(\tilde{r}) \cdot \hat{n} \times \int_{S_{n}} d \bar{r}^{\prime} \bar{b}_{n}\left(\bar{r}^{\prime}\right) \times \nabla^{\prime} g\left(\bar{r}, \bar{r}^{\prime}\right),
$$

where $\bar{t}_{m}$ and $\bar{b}_{n}$ represent the testing and basis functions, respectively. In order to observe the inaccuracy of the MFIE, Figure l(a) shows a scattering problem that involves a conducting cube with $\lambda / 2$ edges. The incident field with a y-polarized electric field propagates in the $-\mathrm{x}$ direction and the RCS is calculated on the $\mathrm{x}-\mathrm{y}$ plane by using both

This work was supported by the Turkish Academy of Sciences in the framework of the Young
Scientist Award Program (LG/TUBA-GEBIP/2002-1-12), and by the Scientific and Technical Research Council of Turkey (TUBITAK) under Research Grant 103E008. 
the EFIE and the MFIE formulations. Three different triangulations are used with the approximate triangle sizes of $\lambda / 10, \lambda / 20$, and $\lambda / 40$. RWG basis functions are used to expand the current while the testing functions are chosen to be the same type in accordance with the Galerkin method.

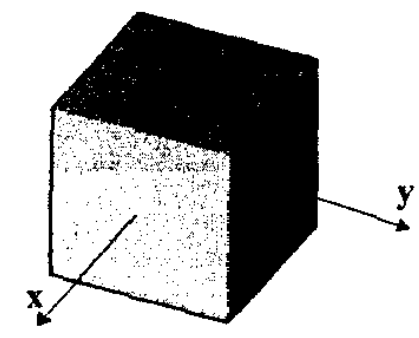

(a)

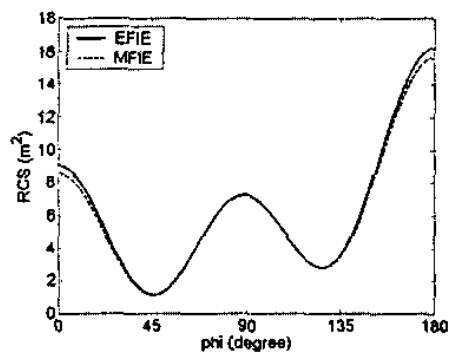

(c)

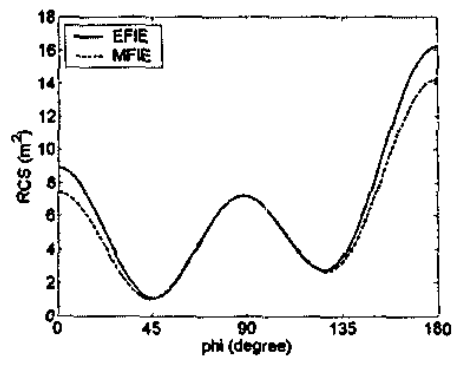

(b)

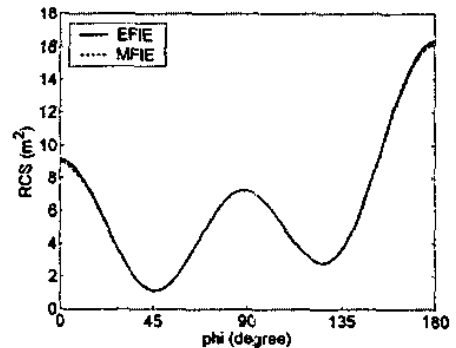

(d)

Figure 1. (a) Conducting cube with $\lambda / 2$ edge, (b) RCS for $\lambda / 10$ triangulation, (c) RCS for $N / 20$ triangulation, (d) RCS for $\lambda / 40$ triangulation.

Figures 1(b), 1(c), and 1(d) show that the change in the RCS values is very limited for the EFIE solution when the mesh size gets smaller. This stipulates that the EFIE converges to a solution even for $\lambda / 10$ discretization. However, the RCS obtained by the MFIE converge to correct level up to $\lambda / 40$, where it matches better the RCS obtained by the EFIE. For the solutions with $\lambda 10$ triangulation, the difference between the RCS curves of two integral equations is significant. The convergence analysis shows that the MFIE is more inaccurate and does not give reliable results with $\lambda / 10$ triangulation.

\section{Investigating the Causes of the Inaccuracy}

We investigated two possible reasons that may lead to inaccuracy of the MFIE:

a) Logarithmic Singularity in the Field Integration: Different from the EFIE, the integration of the field on the testing triangles may involve a logarithmic singularity in 
the MFIE formulation. This singularity is observed for the interactions between the neigbboring triangles and does not cause problems if the testing and basis triangles are on the same plane. However, if the neighboring triangles are on different planes as shown in Figure 2, the singular field is to be included in the interaction and this leads to numerical problems if the field is desired to be tested on the edge of the basis triangle.

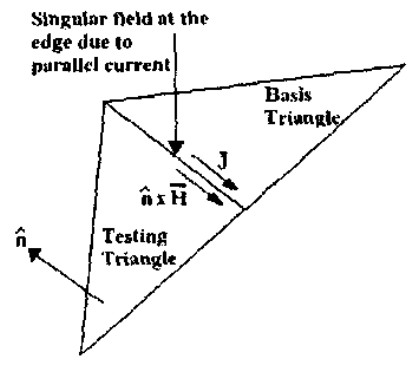

Figure 2. Logarithmic singularity.

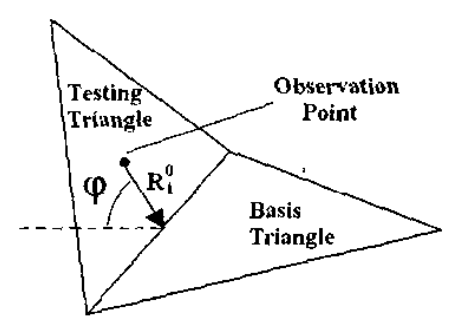

Figure 3, Limit case

Since the singularity is logarithmic towards the edge of the basis triangle, it is possible to integrate the field by taking the sample points strictly inside the testing triangle (not on the edges) and using a sufficient number of points to perform the numerical integration. Experiments show that the application of Gaussian quadrature rule with 32 points is sufficient to calculate the neighboring interactions with less than $1 \%$ error. Alternatively, the integral over the basis integral can be transformed to a line integral around the triangle and the singularity can be avoided by changing the order of the integrations over the basis and testing triangles [6]. Finally, another method, which applies a singularity extraction for the testing integral, can also be used to calculate the neighboring interactions without any problem of the singularity. On the other hand, improvements in the calculation of the MFIE interactions do not solve the accuracy problem of the MFIE.

b) Solid Angle Expression in the MFIE: The limit value of the integral in the MFIE depends on the external solid angle of the surface at the observation point. Equation (I) can be rewritten as

$$
\frac{\Omega_{0}}{4 \pi} \bar{J}(\bar{r})-\hat{n} \times \int_{s^{\prime}(P r)} d \bar{r}^{\prime} \widetilde{J}\left(\bar{r}^{\prime}\right) \times \nabla^{\prime} g\left(\bar{r}, \bar{r}^{\prime}\right)=\hat{n} \times \bar{H}^{i n c}(\bar{r}),
$$

where $\Omega_{0}$ is the external solid angle at the observation point and PV denotes the Cauchy principle value integration. If the observation points are chosen inside the testing triangle, Equation (3) is used with $\Omega_{0}=2 \pi$, since the surface is planar. Therefore, in an MFIE implementation with testing points inside the triangles, the value of $\Omega_{0}$ is always $2 \pi$ as if the gcometry is completely planar. This may lead to the question of whether $\Omega_{0}$ should be chosen to reflect the curvature of the geometry at the testing point. Such a heuristic approach is proposed in [5] as a possible remedy to the inaccuracy of the MFIE. However, not only that the proposed heuristic approach [5] does not guarantee an improvement for all cases, but also it can be rigorously proven that such a guesswork on $\Omega_{0}$ is not necessary at all. This is because a careful investigation of the formulas shows 
that the solid angle is already included in the calculations by means of the interactions between the neighboring triangles. If the formula in [6] is corrected as

$$
\lim _{R_{i}^{0} \rightarrow 0} \beta_{i}=2 \varphi-\pi,
$$

it can be shown that

$$
\int_{S^{\prime}} d \bar{r}^{\prime} \frac{z}{R^{3}}=2 \varphi
$$

when the observation point approaches the basis triangle as shown in Figure 3 . This integral is a part of the scattered field due the basis function and includes the angular dependence in Equation (3). Thus, there is no need to choose or guess the value of $\Omega_{0}$ explicitly.

\section{Conclusion}

MFIE can be shown to give more inaccurate results as compared to the EFIE for the solution of electromagnetic scattering problems with RWG functions. This inaccuracy is more evident when the problem involves geometries with sharp edges and tips. The investigation reported in this paper eliminates some of the possibilities that can be considered as the causes of the MFIE inaccuracy. There are other possible causes to consider, such as the quality of the current modeling based on the choice of the basis functions. Indeed, it can be shown that the accuracy of the MFIE is more dependent on the choice of the basis function than the EFIE, and that the inaccuracy of the MFIE can be controlled with the choice of basis functions [8]

\section{References}

[1] R. F. Harrington, Field Computation by Moment Methods. IEEE Press, 1993.

[2] R. Coifman, V. Rokhlin, and S. Wandzura, "The fast multipole method (FMM) for the wave equation: a pedestrian prescription," IEEE Ant. Propag. Mag., vol. 35, no. 3, pp. 7-12, June 1993.

[3] S. M. Rao, D. R. Wilton, and A. W. Glisson, "Electromagnetic scattering by surfaces of arbitrary shape," IEEE Trans. Antennas Propagat., vol. AP-30, pp. 409-418, May 1982.

[4] R. E. Hodges and Y. Rahmat-Samii, "The evaluation of MFIE integrals with the use of vector triangle basis functions," Micro. Opt. Tech. Lett., vol. 14, no. 1, pp. 9-14, Jan. 1997.

[5] J. M. Rius, E. Úbeda, and J. Parrón, "On the testing of the magnetic field integral equation with RWG basis functions in method of moments," IEEE Trans. Antennas Propagat. Vol. 49, no 11, pp. 1550-1553, Nov. 2001.

[6] Pasi Ylä-Oijala and Matti Taskinen, "Calculation of CFIE impedance matrix elements with RWG and $\mathrm{x}$ RWG Functions," IEEE Trans. Antennas Propagat. Vol. 51, no. 8, pp. 1837-1845, Aug. 2003.

[7] R. D. Graglia, "On the numerical integration of the linear shape functions times the 3-D Green's function or its gradient on a plane triangle," IEEE Trans. Antennas Propagat. Vol. 41, no. 10, pp. 1448-14\$4, Oct. 1993.

[8] Ö. Ergül and L. Gürel, "Improving the Accuracy of the MFIE with the Choice of Basis Functions," 2004 IEEE AP-S International Symposium and URSI Radio Science Meeting, Monterey, CA, June 2004. 